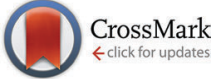

Cite this: J. Mater. Chem. C, 2015 3, 10467

Received 28th May 2015, Accepted 9th July 2015

DOI: $10.1039 / c 5 t c 01535 a$

www.rsc.org/MaterialsC

\section{Thermoelectric transport and microstructure of optimized $\mathrm{Mg}_{2} \mathrm{Si}_{0.8} \mathrm{Sn}_{0.2} \dagger$}

\author{
J. de Boor, $\ddagger^{\star^{a}}$ S. Gupta, $\ddagger^{a}$ H. Kolb, ${ }^{a}$ T. Dasguptab and E. Müller ${ }^{a c}$
}

\begin{abstract}
Solid solutions of magnesium silicide and magnesium stannide exhibit excellent thermoelectric properties due to favorable electronic band structures and reduced thermal conductivity compared to the binary compounds. We have optimized the composition $\mathrm{Mg}_{2} \mathrm{Si}_{0.8} \mathrm{Sn}_{0.2}$ by $\mathrm{Sb}$ doping and obtained a thermoelectric figure of merit close to unity. The material comprises of several phases and exhibits intrinsic nanostructuring. Nevertheless, the main features of electronic transport can be understood within the framework of a single parabolic band model. Compared to $\mathrm{Mg}_{2} \mathrm{Si}$ we observe a comparable power factor, a drastically reduced thermal conductivity and an increased effective mass.
\end{abstract}

\section{Introduction}

Thermoelectric materials can be used to convert (waste) heat directly into electrical energy. They can thus power autonomous devices or enhance the energy efficiency of various applications. ${ }^{1}$ A relatively new approach is the use of thermoelectric materials as thermopower wave based energy sources. Such devices derived from nanoscale thermoelectric materials and chemical fuels employ high energy charge carriers in the non-equilibrium state to create considerable voltages and electrical power and may find application as miniature power sources. ${ }^{2,3}$ The efficiency of the heat to electrical energy conversion is linked to the thermoelectric figure of merit of the materials, given by $Z T=\frac{\sigma S^{2}}{\kappa} T$. Here $\sigma$ is the electrical conductivity, $\kappa$ the thermal conductivity, $S$ the Seebeck coefficient, and $T$ the absolute temperature. $\mathrm{Mg}_{2} \mathrm{Si}$ based solid solutions are among the most promising thermoelectric materials. For this material class, a highly desirable $Z T>1$ has repeatedly been reported ${ }^{4-9}$ and the constituting elements are abundant and non-toxic. $\mathrm{Mg}_{2} \mathrm{Si}$ based solid solutions are therefore efficient and environmentally compatible alternatives to $\mathrm{PbTe}$ and skutterudites. Due to their very low density they are especially attractive for airborne or mobile applications where the weight is crucial.

So far, most of the research studies are dedicated either to pure $\mathrm{Mg}_{2} \mathrm{Si}$ due to its simplicity and the relatively high thermal

\footnotetext{
${ }^{a}$ Institute of Materials Research, German Aerospace Center, Linder Höhe, 51147 Köln, Germany. E-mail: Johannes.deboor@dlr.de

${ }^{b}$ Department of Metallurgical Engineering and Materials Science, Indian Institute of Technology Bombay, Mumbai 400076, India

${ }^{c}$ Institute for Inorganic and Analytical Chemistry, Justus-Liebig-Universität Gießen, Heinrich-Buff-Ring 58, 35392 Gießen, Germany

$\dagger$ Electronic supplementary information (ESI) available. See DOI: 10.1039/ c5tc01535a

\$ Contributed equally.
}

stability or to Sn-rich compositions similar to $\mathrm{Mg}_{2} \mathrm{Si}_{0.4} \mathrm{Sn}_{0.6}$. The latter composition exhibits a crossing of the Si and Sn subbands, which increases the band degeneracy and therefore drastically increases the effective mass of the electrons. ${ }^{4,5,10}$ Furthermore, the thermal conductivity is reduced compared to the binary compositions due to additional alloy scattering. There are only a few reports on the Si-rich side of the $\mathrm{Mg}_{2}(\mathrm{Si}, \mathrm{Sn})$ family. Tani et al. optimized the carrier concentration for $\mathrm{Mg}_{2} \mathrm{Si}_{1-x} \mathrm{Sn}_{x}$ for $x=0.05$ and 0.1 , reaching a $Z T_{\max }$ of 0.68 at $864 \mathrm{~K} .{ }^{11}$ Liu et al. and Samunin et al. reported some transport data for $x=0.2$ and showed a maximum $Z T$ of around 0.8 , but did not present a conclusive optimization with respect to the carrier concentration. ${ }^{5,12}$

However, investigation and optimization of Si-rich $\mathrm{Mg}_{2}(\mathrm{Si}, \mathrm{Sn})$, in particular $\mathrm{Mg}_{2} \mathrm{Si}_{0.8} \mathrm{Sn}_{0.2}$, is very interesting both from a fundamental and an applied point of view. $\mathrm{Mg}_{2} \mathrm{Si}_{0.8} \mathrm{Sn}_{0.2}$ has a significantly lower density than $\mathrm{Mg}_{2} \mathrm{Si}_{0.4} \mathrm{Sn}_{0.6}\left(2.3 \mathrm{~g} \mathrm{~cm}^{-3}<3 \mathrm{~g} \mathrm{~cm}^{-3}\right)$, i.e. for applications where weight is a crucial factor, it might be the optimal choice, even with inferior thermoelectric performance. Moreover, as $\mathrm{Mg}_{2} \mathrm{Si}$ is thermally and chemically more stable than $\mathrm{Mg}_{2} \mathrm{Sn}$ it is plausible that Si-rich compositions are more stable than Sn-rich compositions, allowing operation at higher temperatures. Furthermore, $\mathrm{Mg}_{2} \mathrm{Si}_{0.8} \mathrm{Sn}_{0.2}$ is closer to the technologically more developed $\mathrm{Mg}_{2} \mathrm{Si}$, where progress in contact development has been reported. ${ }^{13,14}$ On the other hand, compared to $\mathrm{Mg}_{2} \mathrm{Si}$ an improvement of the thermoelectric properties can be expected due to increased phonon alloy scattering.

Beyond this, the composition $\mathrm{Mg}_{2} \mathrm{Si}_{0.8} \mathrm{Sn}_{0.2}$ is also very interesting with respect to fundamental aspects. According to the literature reports, there is a miscibility gap between $\mathrm{Mg}_{2} \mathrm{Si}$ and $\mathrm{Mg}_{2} \mathrm{Sn}$ whose exact borders are disputed. ${ }^{4,15-17}$ According to ref. $16, \mathrm{Mg}_{2} \mathrm{Si}_{0.8} \mathrm{Sn}_{0.2}$ is within the miscibility gap. This provides the chance for an intrinsic nanostructure within the material, 
decreasing the thermal conductivity and thus enhancing the thermoelectric performance. This strategy has successfully been employed, e.g. for the PbTe family and half-Heuslers. ${ }^{18,19}$ Modeling of the $\mathrm{Mg}_{2}(\mathrm{Si}, \mathrm{Sn})$ family is important for a thorough understanding and further optimization. This modeling is often performed using a linear interpolation between $\mathrm{Mg}_{2} \mathrm{Si}$ and $\mathrm{Mg}_{2} \mathrm{Sn}$ for parameters like band gaps or interaction potentials. ${ }^{4,20,21}$ These theoretical assumptions have to be validated by experimental results.

In this work, we have therefore studied the microstructure and the thermoelectric properties of $\mathrm{Mg}_{2} \mathrm{Si}_{0.8} \mathrm{Sn}_{0.2}$. We demonstrate charge carrier density optimization by means of $\mathrm{Sb}$ doping. High temperature measurements of the electrical and thermal conductivity, Seebeck coefficient as well as Hall carrier density and mobility reveal that the electronic properties can be modelled reasonably well in the framework of a single parabolic band model. The experimental and the modeling results provide fundamental transport parameters like the effective mass, carrier mobility and interaction potentials. Additionally, we compare our results with data from the binary compound $\mathrm{Mg}_{2} \mathrm{Si}$ and provide insights into the effect of $\mathrm{Si} / \mathrm{Sn}$ substitution on the electronic band structure.

\section{Experimental}

The $\mathrm{Mg}_{2} \mathrm{Si}_{0.8-y} \mathrm{Sn}_{0.2} \mathrm{Sb}_{y}$ ingot material was synthesized by direct melting of the elements in an induction furnace as described in ref. 22. $\mathrm{Sb}$ is a well-known n-type dopant for $\mathrm{Mg}_{2}(\mathrm{Si}, \mathrm{Sn})$ and was added to tune the carrier concentration. To compensate for the loss of $\mathrm{Mg}$ due to evaporation during the melting process, $5 \%$ excess was added. The ingot material was crushed in air using an agate mortar and pestle. Pellets with a diameter of $15 \mathrm{~mm}$ and a height of roughly $1 \mathrm{~mm}$ were obtained by compaction at $850{ }^{\circ} \mathrm{C}$ using current assisted sintering. Further sintering details can be found in ref. 23. The electrical conductivity $\sigma$ and Seebeck coefficient $S$ of the samples were measured concurrently using a custom-built setup. The setup and details of the data analysis can be found elsewhere. ${ }^{24,25}$ The thermal conductivity $\kappa$ of the samples was determined using $\kappa=D \rho c_{\mathrm{p}}$, where the thermal diffusivity $D$ was measured using a commercial LFA-setup (Netzsch) and the density $\rho$ using Archimedes' method. The Dulong-Petit value was used for specific heat $c_{\mathrm{p}}$ calculation. The Hall carrier concentration $n_{\mathrm{H}}$ and mobility $\mu_{\mathrm{H}}$ were determined in a van der Pauw geometry using a custom-built setup under variable magnetic field. ${ }^{26,27}$ The XRD data were obtained using a Siemens D5000 and Rietveld refinement of the lattice parameter $a$ was performed using Topas 4.2. The SEM images were taken using a Zeiss Ultra 55 equipped with an EDX detector. Measurement uncertainties are 5\% for $\sigma$ and $S, 8 \%$ for $\kappa$ and $15 \%$ for the carrier concentration and mobility. These result in a total uncertainty of $12 \%$ for the thermoelectric figure of merit if we sum the errors squared and assume that they are independent of each other and $23 \%$ as the worst case scenario. Also note that here the total measurement uncertainty is given, whereas the reproducibility is usually better by a factor of 2 .

\section{Results}

Table 1 shows the nominal composition of five synthesized samples as well as the measured densities $\rho$. Densities have been obtained using the sample geometry and weight as well as by employing Archimedes' principle. Comparing both methods the geometrical density $\rho_{\text {geo }}$ has the higher uncertainty; however the Archimedes density $\rho_{\mathrm{A}}$ tends to overestimate the density in principle as it does not account for open porosity in the material. The results for both densities are similar within the experimental errors, except for sample \#3. This indicates that there is little open porosity in the samples. The density can be used to estimate the Sn content $x$ of the samples: $x \approx \frac{\rho-\rho_{\mathrm{Mg}_{2} \mathrm{Si}}}{\rho_{\mathrm{Mg}_{2} \mathrm{Sn}}-\rho_{\mathrm{Mg}_{2} \mathrm{Si}}}$. We used the Archimedes density for this calculation as the geometrical density is prone to underestimate the Sn content in the case of open porosity in the samples. The thus calculated $\mathrm{Sn}$ content is lower than the nominal content of $x=0.2$, but shows some increase with increase in the $\mathrm{Sb}$ concentration.

Fig. 1 shows the XRD results. All major peaks can be indexed according to the reported anti-fluorite structures (space group $F m \overline{3} m$ ) of $\mathrm{Mg}_{2} \mathrm{Si}$ and $\mathrm{Mg}_{2} \mathrm{Sn}$. The minor peak at $2 \theta \approx 43^{\circ}$ corresponds to $\mathrm{MgO}$, an impurity often observed in this material class. ${ }^{28}$ The zoom-in around the 220 peak in Fig. 1(b) shows that the peaks are relatively broad and show a shift towards smaller angles, i.e. larger lattice constants compared to $\mathrm{Mg}_{2} \mathrm{Si}$.

The relationship between the lattice constant $a$ and Sn content $x$ is approximately given by $x=\left(a-a_{\mathrm{Mg}_{2} \mathrm{Si}}\right) /(0.0427 \mathrm{~nm}) .{ }^{20}$ The calculated values of $x$ are given in Table 1 and confirm the results and trends obtained from the density data. In fact, the good agreement between the Sn content obtained from density data and the XRD peak shift indicates good compaction and a high relative density of the samples, as significant (closed) porosity leads to a reduced $x$ from the density data but does not affect that from the XRD peak shift.

Microstructural analysis by SEM shows a multiphase sample with a matrix in grey and minor phases in light grey and dark grey, as shown in Fig. 2. The image is taken for sample \#5, but the microstructure is similar in all samples, see Fig. S2-S4 (ESI $\dagger$ ). EDX analysis of all samples reveals that the matrix and the minor phases do not have sharp compositions, but consist of domains with similar, yet distinct local compositions.

The Sn content $x$ and the approximate phase fractions are given in Table 2. Employing the distinct grey values of the three phases, the graphical analysis software ImageJ has been used to

Table 1 Sample properties: nominal compositions, densities, and Sn content $x$ calculated from density and XRD data

\begin{tabular}{llllll}
\hline Nominal composition & & $\begin{array}{l}\rho_{\text {geo }} \\
{\left[\mathrm{g} \mathrm{cm}^{-3}\right]}\end{array}$ & $\begin{array}{l}\rho_{\mathrm{A}} \\
{\left[\mathrm{g} \mathrm{cm}^{-3}\right]}\end{array}$ & $x$ from $\rho_{\mathrm{A}}$ & $\begin{array}{l}x \text { XRD } \\
\text { XRom }\end{array}$ \\
\hline $\mathrm{Mg}_{2} \mathrm{Si}_{0.8} \mathrm{Sn}_{0.2}$ & $\# 1$ & 2.17 & 2.21 & 0.132 & 0.128 \\
$\mathrm{Mg}_{2} \mathrm{Si}_{0.795} \mathrm{Sn}_{0.2} \mathrm{Sb}_{0.005}$ & $\# 2$ & 2.25 & 2.24 & 0.145 & 0.140 \\
$\mathrm{Mg}_{2} \mathrm{Si}_{0.79} \mathrm{Sn}_{0.2} \mathrm{Sb}_{0.01}$ & $\# 3$ & 2.12 & 2.21 & 0.128 & 0.117 \\
$\mathrm{Mg}_{2} \mathrm{Si}_{0.785} \mathrm{Sn}_{0.2} \mathrm{Sb}_{0.015}$ & $\# 4$ & 2.33 & 2.31 & 0.174 & 0.170 \\
$\mathrm{Mg}_{2} \mathrm{Si}_{0.78} \mathrm{Sn}_{0.2} \mathrm{Sb}_{0.02}$ & $\# 5$ & 2.29 & 2.31 & 0.168 & 0.170
\end{tabular}



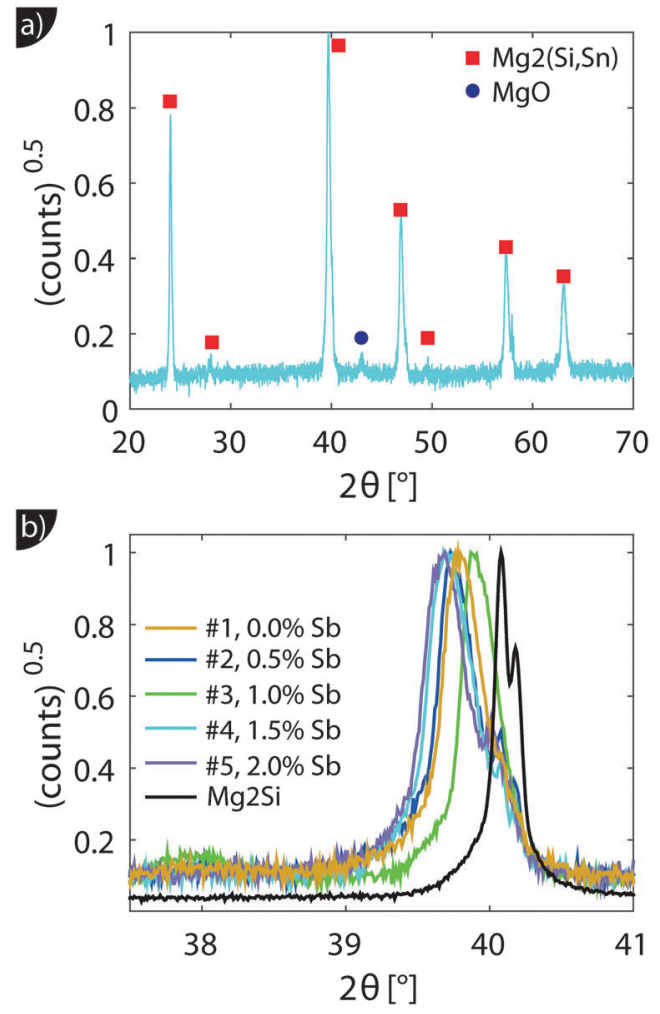

Fig. 1 (a) X-ray diffraction data of sample \#2. All major peaks can be indexed as (shifted) $\mathrm{Mg}_{2} \mathrm{Si}$ peaks. Minor peaks for $\mathrm{MgO}$ can also be observed. (b) The zoom-in around the (220) peak reveals peak broadening and emphasizes the shift compared to the $\mathrm{Mg}_{2} \mathrm{Si}$ sample.

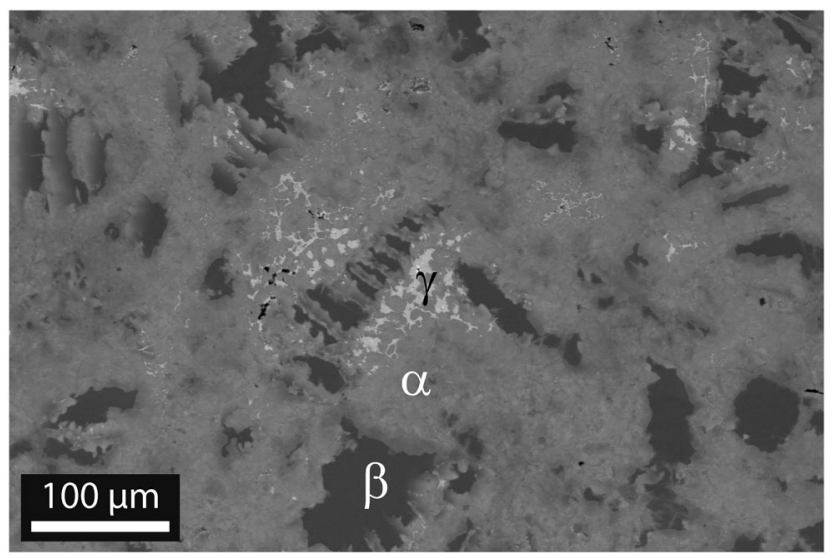

Fig. 2 Backscattered image of sample \#5. Three different phases are clearly distinguishable: the main phase $\alpha$, the $\mathrm{Mg}_{2} \mathrm{Si}$-like phase $\beta$ and the Sn-rich phase $\gamma$. EDX analysis reveals that these phases show significant local concentration fluctuations.

estimate the (areal) fraction of each phase. It yields $85 \%$ main phase, $13 \%$ of the $\mathrm{Mg}_{2}$ Si-like phase and about $2 \%$ for the Sn-rich phase for the two-dimensional image. To calculate the $3 \mathrm{D}$ values, we assumed that the main phase is the matrix and the minor phases are isotropically included within the matrix. The phase fraction for the minor phases is then given by $z_{2 \mathrm{D}}{ }^{3 / 2}$, where $z_{2 \mathrm{D}}$ is the fraction in the two dimensional image.
Table 2 Sn content $x$ and phase fraction of a typical $\mathrm{Mg}_{2} \mathrm{Si}_{0.8} \mathrm{Sn}_{0.2}$ sample

\begin{tabular}{|c|c|c|c|}
\hline & $\begin{array}{l}\text { Main phase } \\
(\alpha)\end{array}$ & $\begin{array}{l}\text { Si-rich phase } \\
(\beta \text {, dark })\end{array}$ & $\begin{array}{l}\text { Sn-rich phase } \\
(\gamma, \text { bright })\end{array}$ \\
\hline $\begin{array}{l}\text { Sn content } x \\
\text { Phase fraction }\end{array}$ & $\begin{array}{l}0.1<x<0.2 \\
0.95\end{array}$ & $\begin{array}{l}x<0.03 \\
0.05\end{array}$ & $\begin{array}{l}0.4<x<0.6 \\
<0.01\end{array}$ \\
\hline
\end{tabular}

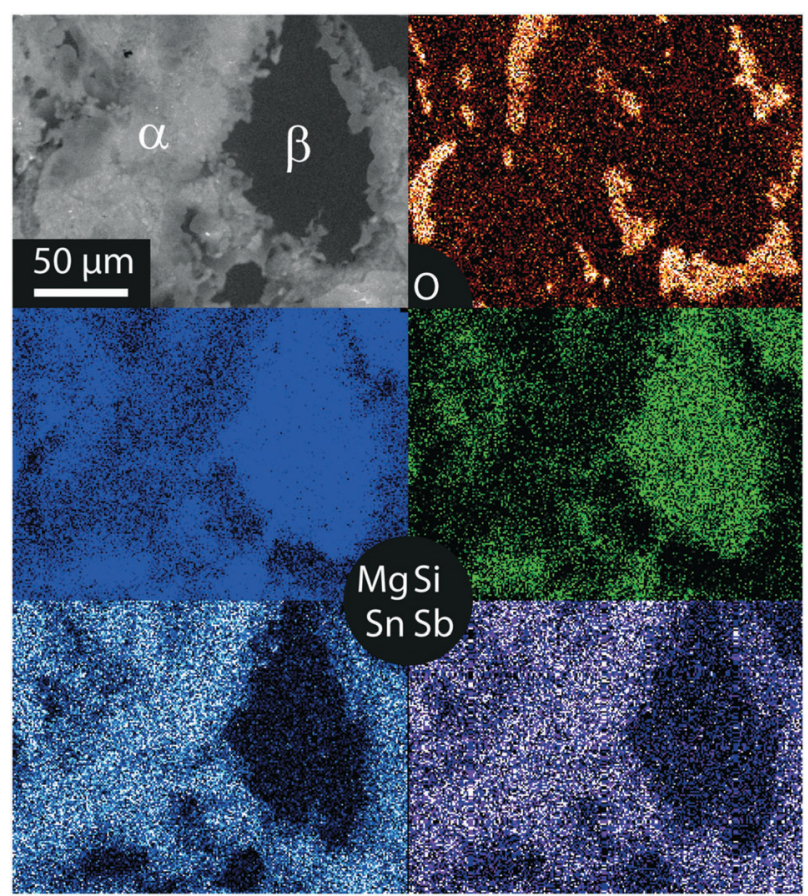

Fig. 3 BSE image and elemental mapping of O, Mg, Si, Sn and Sb.

Fig. 3 shows the element mapping of a typical $\mathrm{Mg}_{2} \mathrm{Si}_{0.8} \mathrm{Sn}_{0.2}$ sample. The $\mathrm{Mg}_{2}$ Si-like and the main phase are clearly distinguishable, and it can also be seen that the matrix phase itself has a spatially varying Sn content. Another interesting feature is the observed difference in the Sb content for the main phase and the $\mathrm{Mg}_{2} \mathrm{Si}$-like phase. $\mathrm{Sb}$ is significantly more dissolved in the main phase with a higher Sn content. Presumably Sb can be more easily incorporated in Sn-richer phases due to their larger lattice constant. The EDX analysis also detects some oxygen, mainly at interface regions between the matrix phase and the $\mathrm{Mg}_{2}$ Si-like phase. As $\mathrm{MgO}$ has been identified by XRD it can be deduced that oxygen is present presumably in the form of $\mathrm{MgO}$. The matrix phase and the $\mathrm{Mg}_{2} \mathrm{Si}$ like phase are also visible in the Mg mapping. As the lattice constant increases with increase in the $\mathrm{Sn}$ content, the $\mathrm{Mg}$ density is higher in the $\mathrm{Mg}_{2} \mathrm{Si}$-like phase than in the matrix phase, which results in the observed contrast.

\section{Transport data}

Despite the microstructure the samples exhibit a good macroscopic homogeneity of their electronic properties as evidenced by a local mapping of the Seebeck coefficient (see Fig. S6, ESI $\dagger$ ).

Transport data of $\mathrm{Mg}_{2} \mathrm{Si}_{0.8-y} \mathrm{Sn}_{0.2} \mathrm{Sb}_{y}$ are shown from room temperature to $740 \mathrm{~K}$ in Fig. 4 . Due to better visibility only the 
thermal conductivity data of the undoped sample (\#1) are shown here; the complete data are presented in Fig. S5 of the ESI. $\dagger$ The electrical conductivity (a) shows the typical decrease with increase in the temperature of a highly doped semiconductor above $400 \mathrm{~K}$; below $400 \mathrm{~K}$ a plateau can be observed for some of the samples. The electrical conductivity also exhibits the expected increase with increase in doping. The Seebeck coefficient (b) decreases with increase in doping and increases approximately linearly with increase in the temperature for all samples.

The thermal conductivity (c) decreases with temperature for all samples but shows a much higher value for the sample with the highest doping. The thermoelectric figure of merit $Z T$ is calculated from (a) to (c) and shows an increase with temperature for all samples. Sample \#4 with $y=0.015$ has the highest thermoelectric figure of merit with $Z T=0.95$ at $740 \mathrm{~K}$. While the $Z T$ values for the less doped samples are lower, but comparable, sample \#5 has a drastically lower figure of merit. Fig. 4(e) reveals a roughly temperature independent carrier density of all samples, except for sample \#5. Here the carrier concentration is roughly constant up to $650 \mathrm{~K}$ after which $n_{\mathrm{H}}$ decreases rapidly. As the sample has been stable during the $S-\sigma$ and LFA measurements beforehand, we do not know what caused the irreversible change in the sample. It can be seen that the carrier density data of the samples are consistent with the results for $S$ and $\sigma$ and that the control over the carrier
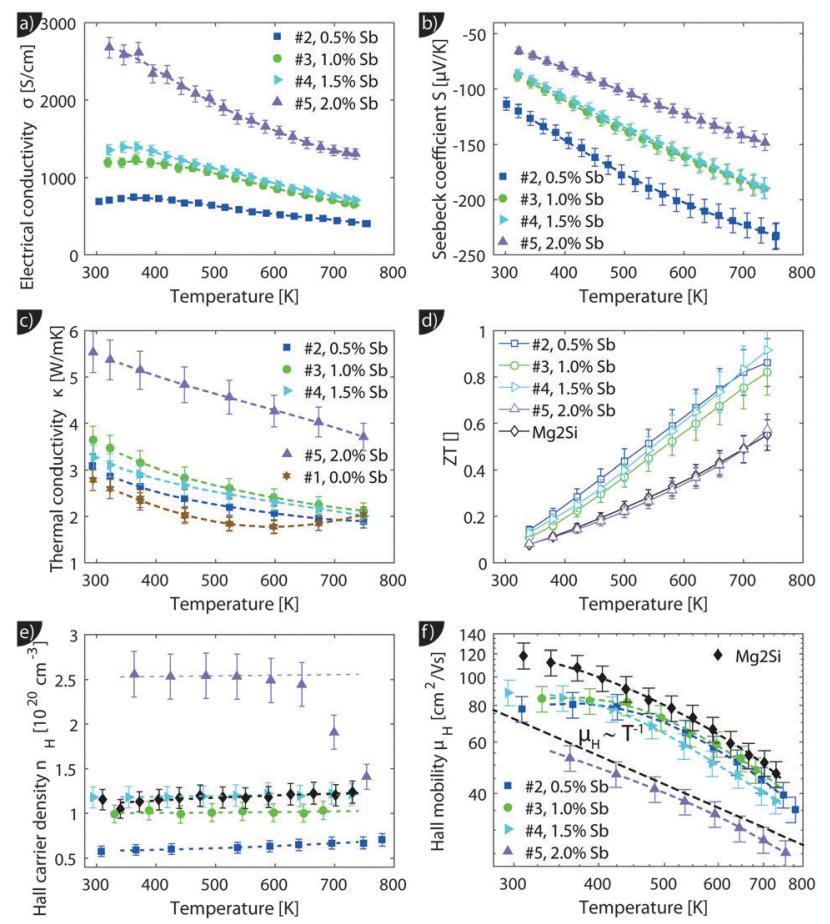

Fig. 4 Transport measurement data for $\mathrm{Mg}_{2} \mathrm{Si}_{0.8-y} \mathrm{Sn}_{0.2} \mathrm{Sb}_{y}$ samples. Full markers indicate measurement data, empty markers the calculated data and the dashed lines the fitted transport data that are used for modeling. The thermoelectric figure of merit (d) as well as the Hall carrier concentration (e) and mobility (f) are also presented for a sample with the composition $\mathrm{Mg}_{2} \mathrm{Si}_{0.9875} \mathrm{Sb}_{0.0125}$ (i.e. without tin) for comparison. This sample is labelled as "Mg $2 \mathrm{Si}^{\prime}$. concentration is not perfect as the actual Hall carrier concentration does not exactly follow the linear trend expected from the nominal doping composition. Doping is relatively effective as one would roughly expect $1.5 \times 10^{20} \mathrm{~cm}^{-3}$ carriers for a nominal composition of $\mathrm{Mg}_{2} \mathrm{Si}_{0.79} \mathrm{Sn}_{0.2} \mathrm{Sb}_{0.01}$ if one carrier per $\mathrm{Sb}$ atom is provided. The Hall mobility $(f)$ decreases with increase in the temperature after an initial plateau for samples \#2-\#4; sample \#5 shows a monotonic trend with significantly lower absolute values.

We will now analyze the results in the framework of a single parabolic band model (SPB). ${ }^{29}$ This model has been employed for $\mathrm{Mg}_{2} \mathrm{Si}$ and $\mathrm{Mg}_{2} \mathrm{Si}_{1-x} \mathrm{Sn}_{x}$ with reasonable success beforehand. ${ }^{\text {,21,30 }}$ For $T>500 \mathrm{~K} \mu_{\mathrm{H}} \propto T^{-p}$ holds with $1<p<1.5$, which indicates acoustic phonon (AP) scattering as the dominant scattering mechanism. At lower temperatures, there is some deviation from this behavior, presumably due to grain boundary scattering of the charge carriers at the interfaces. ${ }^{28}$ A possible influence of alloy scattering cannot be excluded, but is not expected to be dominant due to the relatively low Sn content. ${ }^{6}$ Grain boundary scattering is an extrinsic scattering mechanism so that AP scattering can assumed to be the dominant intrinsic mechanism at all temperatures. In this case, the reduced chemical potential $\eta$ and the DOS effective mass $m^{*}$ of the electrons can be calculated using

$$
S=-\frac{k}{e}\left(\frac{2 F_{1}(\eta)}{F_{0}(\eta)}-\eta\right),
$$

and

$$
n=4 \pi\left(\frac{2 m^{*} k_{\mathrm{B}} T}{h^{2}}\right)^{3 / 2} F_{1 / 2}(\eta) .
$$

Here $e$ is the elementary charge, $k_{\mathrm{B}}$ the Boltzmann's constant, $F_{i}$ the Fermi integral of order $i$, and the reduced chemical potential $\eta$ is given by $\eta=\frac{E_{\mathrm{F}}}{k_{\mathrm{B}} T}$. The measured Hall carrier density $n_{\mathrm{H}}$ is linked to the true carrier density by $n_{\mathrm{H}}=n / r_{\mathrm{H}}$ with the Hall scattering factor given by $r_{\mathrm{H}}=\frac{1.5 F_{0.5} F_{-0.5}}{2 F_{0}{ }^{2}}$. The chemical potentials for samples \#2-\#5 are plotted in Fig. 5(a). It can be seen that the chemical potential lies above the conduction band edge at room temperature for all samples. It decreases with increase in the temperature and crosses the band edge for the lowest doped sample.

The effective mass increases with temperature for all samples. While the three lower doped samples increase roughly from $1 m_{0}$ to $1.25 m_{0}$ in the measured temperature range, the sample with the highest doping increases from $1.25 m_{0}$ to $1.35 m_{0} ; m_{0}$ is the free electron mass. The Pisarenko plot in Fig. 5(c) shows reasonable agreement between the experimental and the modeling data.

The lattice thermal conductivity (and the bipolar contribution) is plotted in Fig. 5(e). It is given by $\kappa_{\text {lat }}+\kappa_{\text {bip }}=\kappa-L \sigma T$, with $L=\frac{k^{2}}{e^{2}} \frac{3 F_{0} F_{2}-4 F_{1}^{2}}{F_{0}^{2}}$ for the SPB model with AP scattering. The thermal conductivities of samples \#1-\#4 are comparable with a slight reduction for increased doping. The lattice 

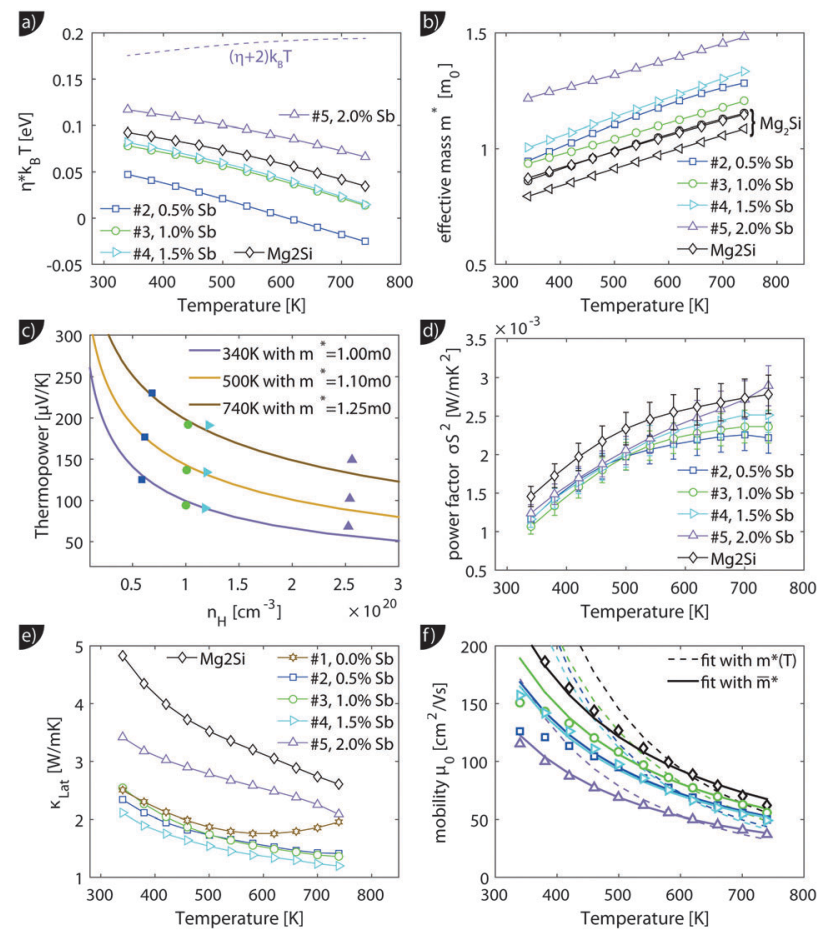

Fig. 5 Results from the single parabolic band model for $\mathrm{Mg}_{2} \mathrm{Si}_{0.8-y} \mathrm{Sn}_{0.2} \mathrm{Sb}_{y i}$ for comparison, the data of $\mathrm{Mg}_{2} \mathrm{Si}_{0.9875} \mathrm{Sb}_{0.0125}$ (" $\mathrm{Mg}_{2} \mathrm{Si}$ ') from ref. 28 are also presented. (a) Calculated chemical potential for all samples. For the sample with the largest carrier concentration (\#5) $(\eta+2) k_{\mathrm{B}} T$ is plotted as well; this gives an impression up to which energy a significant number of carriers are excited. (b) All samples show an increase in the effective mass with increase in the temperature, with sample \#5 having a significantly higher effective mass. (c) The Pisarenko plot shows decent agreement between experimental data and theoretical curves using the temperature dependent effective masses of (b). ( $d$ and e) Power factor $\sigma S^{2}$ and lattice thermal conductivity (overlaid by bipolar contribution). Compared to $\mathrm{Mg}_{2} \mathrm{Si}$, the $\mathrm{Mg}_{2} \mathrm{Si}_{0.8} \mathrm{Sn}_{0.2}$ samples show a slightly reduced power factor but a drastically reduced lattice thermal conductivity. (f) A fit of the mobility parameter $\mu_{0} v s$. $T$ can be used to obtain the deformation potential. Dashed lines show the results using $m^{\star}(T)$ while the solid lines the results from an averaged effective mass.

thermal conductivity follows the power law $\kappa_{\text {lat }}=A+B / T^{p}$ with $-1<p<-0.5$. Umklapp phonon scattering predicts a $\kappa_{\text {lat }} \propto T^{-1}$ behavior, while $\kappa_{\text {lat }} \propto T^{-0.5}$ corresponds to alloy scattering as the dominant phonon scattering mechanism. The measurement results therefore indicate a mixed scattering mechanism. At high temperature, the onset of the bipolar contribution is clearly visible for the undoped sample. The sample with the highest doping has a lattice thermal conductivity, which is more than $50 \%$, higher than the others, indicating a significantly different thermal transport in this sample.

The carrier density independent mobility $\mu_{0}$ is plotted in Fig. 5(f) and is related to the Hall mobility by ref. 29. It is a material parameter and is thus supposed to be independent of the carrier concentration. Indeed one notices that the mobilities for samples \#2-\#4 are very similar. The mobility of sample \#5 is significantly lower; however, the difference is not as large as for $\mu_{\mathrm{H}}$. The mobility data can be used to extract a further material parameter, the deformation potential $E_{\mathrm{def}}$ which quantifies the

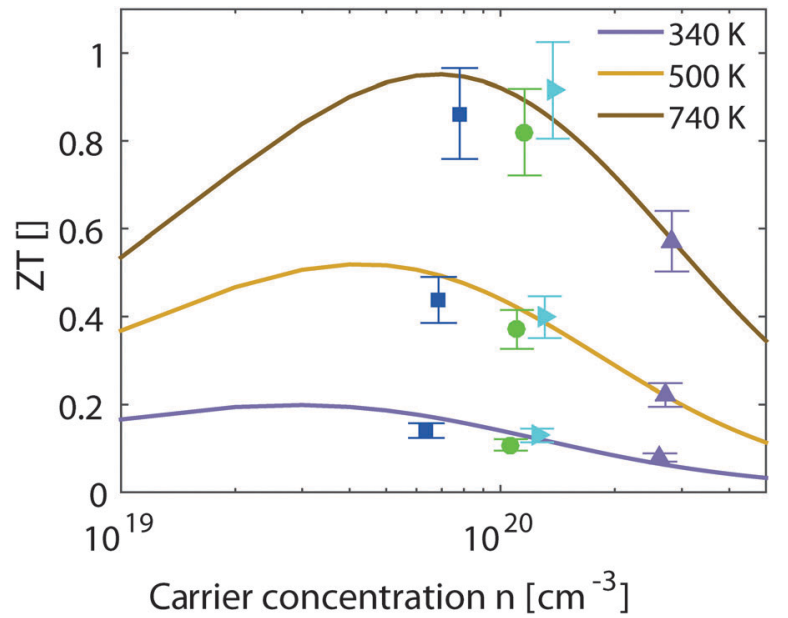

Fig. 6 Experimental and theoretical results for the thermoelectric figure of merit vs. the carrier concentration at different temperatures.

interaction between acoustic phonons and charge carriers. It is given by ref. 31 :

$$
\mu_{0}=\frac{e \pi \hbar^{4}}{\sqrt{2}(k T)^{1.5}} \frac{1}{\left(m_{i}\right)^{2.5}} \times \frac{C_{11}}{E_{\mathrm{def}^{2}}}=A(T) \times \frac{C_{11}}{E_{\mathrm{def}^{2}}} \times \frac{1}{T^{1.5}} .
$$

Fig. 5(f) shows fits of eqn (3) to the experimental data using $m^{*}(T)$, as shown in Fig. 5(b) (dashed lines), and an average effective mass that is constant with temperature (full lines).

The thermoelectric potential of the material can be estimated by calculating $Z T(n, T)$. The basic equation can be rearranged as

$$
Z T=\frac{S^{2}}{L+(\psi \beta)^{-1}}
$$

with

$$
\psi=\frac{8 \pi e}{3}\left(\frac{2 m k_{\mathrm{B}}}{h^{2}}\right)^{1.5} F_{0}, \quad \beta=\mu_{0}\left(\frac{m^{*}}{m}\right)^{1.5} T^{2.5} / \kappa_{1},
$$

and $\mu_{0}=\mu_{\mathrm{H}} \times 2 F_{0} / F_{-0.5}$. The material parameter $\beta$ is plotted in Fig. S7d (ESI $\dagger$ ). For the theoretical curves shown in Fig. 6, the average $\beta$ of samples $\# 2-\# 4$ has been employed.

The results from eqn (4) show the expected trends: an increase in $Z T_{\max }$ with increasing temperature and a shift of the optimal carrier concentration towards higher values for increasing temperature. Our experimental data show good agreement with the modeling results. $Z T_{\text {max }}$ obtained both from the SPB model and the experimental data is $\approx 0.95$ at $740 \mathrm{~K}$. The best experimental value is at $n_{\mathrm{opt}}=1.2 \times 10^{20} \mathrm{~cm}^{-3}$, while the model predicts $0.7 \times 10^{20} \mathrm{~cm}^{-3}$; however, the maximum is relatively broad and the model does not account for differences in $\kappa_{\text {lat }}$ observed between the samples.

\section{Discussion}

\section{Microstructure}

The XRD and density measurements confirm the successful synthesis of $\mathrm{Mg}_{2} \mathrm{Si}_{1-x-y} \mathrm{Sn}_{x} \mathrm{Sb}_{y}$ with $x \approx 0.15$. This is slightly smaller than that expected from the initial weight stoichiometry. We suspect that some elemental Sn is lost during the synthesis process. The Sn content values obtained from the XRD peak shift 
and from Archimedes density agree well with each other and show the same minor increase of $x$ with increase in the dopant concentration. It is well known from other material classes (e.g. $\mathrm{CoSb}_{3}$ type skutterudites ${ }^{32}$ ) that small changes in the composition can influence the solubility of one of the components in the material. This is plausible in this case because the targeted composition is close to the edge of the reported miscibility gap. The electron microscope investigations reveal multiphase samples composed of three different phases. However, these phases are not homogeneous but consist of domains with a similar but distinct stoichiometry. The main phase has a Sn content of $0.1<x<0.2$ and comprises of around $95 \%$ of the material volume. The second phase with $5 \mathrm{vol} \%$ is $\mathrm{Mg}_{2} \mathrm{Si}$-like with $x<0.03$. The third phase has $<1$ vol\% and $0.4<x<0.6$. These observations are in agreement with the reported miscibility gap between $\mathrm{Mg}_{2} \mathrm{Si}$ and $\mathrm{Mg}_{2} \mathrm{Sn}$. Our results are also in quantitative agreement with the data from ref. 16 taking into account that EDX analysis gives a lower limit for $x$ at the left side of the miscibility gap and an upper limit for the right side due to the limited spatial resolution.

Our SEM/EDX results confirm the XRD results where the observed broad peaks indicate stoichiometric variations. The observed minor phases can very well be hidden in the shoulders of the broad peaks. EDX mapping also shows a lower content of the dopant $\mathrm{Sb}$ in the $\mathrm{Mg}_{2} \mathrm{Si}$-like phase compared to the matrix phase. As the matrix phase has a slightly larger lattice constant a better Sb solubility can be expected.

\section{Transport data}

We have obtained $Z T_{\max }=0.95$ at $740 \mathrm{~K}$, higher than previous reports on the same composition. Liu et al. and Samunin et al. both obtained a $Z T_{\max }$ around 0.8. ${ }^{5,12}$ The samples investigated by Liu et al. exhibit a slightly higher power factor than our samples but a significantly higher thermal conductivity. Tani et al. studied the composition $\mathrm{Mg}_{2} \mathrm{Si}_{0.9} \mathrm{Sn}_{0.1}$ and obtained a $Z T_{\text {max }}=0.68$ at $864 \mathrm{~K}$. Comparing our transport data with the work of Tani et al. on $\mathrm{Mg}_{2} \mathrm{Si}_{0.9} \mathrm{Sn}_{0.1}$, we find similar power factors but a higher lattice thermal conductivity in their work. ${ }^{11}$ A possible explanation is the intrinsic nanostructuring in our samples due to the existence of three distinct phases as well as the compositional variations within these phases. This characteristic has not been discussed in the previous reports; however, as no details on the microstructure are given in these reports a thorough comparison is difficult. We also note that our lattice thermal conductivities for samples \#1-\#4 are in good agreement with the experimental and theoretical results obtained at room temperature by Zaitsev et $a l^{33}$ The significantly higher thermal conductivity of $\# 5$ has to be related to the microstructure of the sample; however, since microstructural investigations have not revealed a fundamental difference between \#5 and \#2-\#4, further investigations are required.

\section{Electronic transport analysis}

We have modeled the electronic transport properties within the SPB model with AP scattering as the dominant scattering mechanism. Overall the agreement of experimental data and
Table 3 SPB parameters. For $m^{\star}$ and $\kappa_{\text {lat }}$, the average values for samples \#2-\#4 are given

\begin{tabular}{llllll}
\hline$T$ & $n_{\text {opt }}\left[10^{19} \mathrm{~cm}^{-3}\right]$ & $Z T_{\max }$ & $m^{*}\left[m_{0}\right]$ & $\kappa_{\text {lat }}\left[\mathrm{W} \mathrm{mK}^{-1}\right]$ & $E_{\text {def }}[\mathrm{eV}]$ \\
\hline 340 & 3 & 0.2 & 1 & 2.3 & 13.0 \\
740 & 7 & 0.95 & 1.25 & 1.3 & 13.0 \\
\hline
\end{tabular}

modeling predictions is good. At low temperatures, the fingerprint of the second scattering mechanism is visible in $\sigma$, which can be related to the observed $\mathrm{MgO}$ in the samples. It has been shown that $\mathrm{MgO}$ can cause additional grain boundary scattering and hence reduce carrier mobility and electrical conductivity. ${ }^{28,34}$ The main parameters used in or extracted from the model are summarized in Table 3.

We found an optimum carrier concentration $n_{\text {opt }}$ which is lower than the value $\left(n_{\mathrm{opt}}=18 \times 10^{19} \mathrm{~cm}^{-3}\right)$ stated by Liu et al. $;^{5}$ however the carrier concentration optimization was mainly performed in view of compositions with a higher Sn content in their work. With respect to the effective mass we found good agreement with the literature reports: Liu et al. obtained $m^{*}=0.93 m_{0}$ for $\mathrm{Mg}_{2} \mathrm{Si}_{0.8} \mathrm{Sn}_{0.2}$, while Tani et al. obtained $m^{*}=0.9 m_{0}$ for $\mathrm{Mg}_{2} \mathrm{Si}_{0.9} \mathrm{Sn}_{0.1}$ at room temperature.

However, there are also deviations from the SPB model. Firstly, we observe an apparent increase of the carrier concentration (see Fig. 4 for $n_{\mathrm{H}}(T)$ or Fig. S7(b) (ESI $\dagger$ ) for $n(T)$ ). Secondly, we found a clear increase in the effective mass with temperature and a difference between the sample with the highest doping (\#5) and the other samples. Thirdly, the carrier density corrected mobility $\mu_{0}$ differs for sample \#5 compared to samples \#2-\#4 (see Fig. 5(f)), although it is a material parameter and is supposed to be independent of carrier concentrations.

The increase in $n$ and $m^{*}$ with $T$ was similarly found in $\mathrm{Mg}_{2} \mathrm{Si}^{28,30}$ and might be due to the non-parabolicity of the bands. The observed difference in $m^{*}$ for \#5 compared to \#2-\#4 could be due to either a non-parabolic band or by the influence of the second conduction band with higher $m^{*}: \mathrm{Mg}_{2} \mathrm{Si}_{1-x} \mathrm{Sn}_{x}$ has two threefold degenerate conduction bands $\mathrm{CB}_{\mathrm{L}}$ and $\mathrm{CB}_{\mathrm{H}}$ with a band gap $E_{0}$ that depends on $x$. For small $x$ the light conduction band is at a lower energy, while for large $x$ the heavy band is at lower energy. The cross-over is around 0.6.,10 The band gap between the two conduction bands is less clear for arbitrary $x$. Zaitsev et al. used $E_{0, x=0}=0.4 \mathrm{eV}$ (with $\mathrm{CB}_{\mathrm{L}}$ closer to the valence band) and $E_{0, x=1}=0.2 \mathrm{eV}$ (with $\mathrm{CB}_{\mathrm{H}}$ closer to the valence band). They suggested a linear interpolation in between which results in $E_{0, x=0.2}=0.28 \mathrm{eV} .^{4}$ Bahk et al. used the same assumption for a recent transport modeling. ${ }^{20}$ On the other hand, Bourgeois et al. calculated $E_{0, x=0}=0.19 \mathrm{eV}$ and $E_{0, x=1}=0.28 \mathrm{eV}$ which correspond to $E_{0, x=0.2}=0.1 \mathrm{eV}$, i.e., a much smaller band gap for the investigated composition. ${ }^{15}$ Tan et al. calculated the interband gaps using DFT for different compositions obtaining $E_{0, x=0.25} \approx 0.3 \mathrm{eV} .^{35}$ The discrepancy in the literature reports shows that the interband gap is not well characterized as the band positions are temperature dependent and the calculations do not account for this.

One possible explanation for the experimentally observed increase in $m^{*}$ for sample \#5 compared to \#2-\#4 is thus a 
contribution of the second conduction band $\mathrm{CB}_{\mathrm{H}}$ to the electronic transport. If $\mathrm{CB}_{\mathrm{H}}$ is within $(\eta+2) k_{\mathrm{B}} T$ some contribution can be expected. Fig. 5(a) indicates that this would be the case for $E_{0}<0.2 \mathrm{eV}$. On the other hand, DFT calculations have shown that the bands of $\mathrm{Mg}_{2}(\mathrm{Si}, \mathrm{Sn})$ are not strictly parabolic and therefore the effective mass depends on the chemical potential. $^{35,36}$ In this case, however, the density-of-states effective mass, which is related to the band shape, is not identical to the momentum effective mass (which controls the transport integrals) anymore. ${ }^{37}$ Considering only the effective mass data can thus not provide clear evidence on $E_{0}$ and the question whether one or two bands contribute. Further insight can be gained by a detailed analysis of the mobility data. The fits of $\mu_{0}(T)$ to extract the electron acoustic phonon interaction parameter $E_{\text {def }}$ are plotted using $m^{*}(T)$, as shown in Fig. 5(f) as dashed lines. The fits are performed in the temperature region where AP scattering is clearly dominant, i.e. above $500 \mathrm{~K}$. The agreement between experimental data and theoretical results is not very good, in particular with respect to the temperature dependence. As the strong temperature dependence of $m^{*}(T)$ is unexpected and might be an artifact of the assumed parabolic band structure we have also fitted the data using the average, temperature independent $m^{*}$ of each sample. The results are plotted in full lines and are in almost perfect agreement with the experimental data above $450 \mathrm{~K}$. This indicates that the observed apparent increase in $m^{*}(T)$ is indeed an artifact of the simple SPB model assumptions.

The phonon deformation potential can be extracted from the fits if the elastic constant $C_{11}$ is known. As this is not the case for this particular composition we have used a linear interpolation of the experimental room temperature values from $\mathrm{Mg}_{2} \mathrm{Si}$ and $\mathrm{Mg}_{2} \mathrm{Sn}$, yielding $C_{11, x=0.2} \approx 110 \mathrm{GPa}^{38,39}$ This is in decent agreement with preliminary data obtained from resonant ultrasonic spectroscopy giving $\approx 100 \mathrm{GPa} .{ }^{40}$ The fitting results for $E_{\text {def }}$ of samples \#2-\#5 are $13.1 \mathrm{eV}, 13.5 \mathrm{eV}, 12.8 \mathrm{eV}$, $12.8 \mathrm{eV}$, respectively, giving an average value of $E_{\mathrm{def}, x=0.2}=13.0 \mathrm{eV}$. The good agreement between the results for each sample (although $\mu_{0}$ is different) firstly increases the credibility of the result for $E_{\mathrm{def}, x=0.2}$ and secondly argues against a significant influence of the second band $\mathrm{CB}_{\mathrm{H}}$ on the electronic transport: as the deformation potential for the two sub-bands differs by more than $50 \%,{ }^{6}$ one should see a difference in $E_{\text {def }}$ between \#5 and samples \#2-\#4. This as well as the much better fits of $\mu_{0}(T)$ for a temperature-independent $m^{*}$ indicate that the observed differences in the mobility parameter $\mu_{0}$ between the samples and the apparent temperature dependent $m^{*}$ are rather the consequences of a not strictly parabolic band $\mathrm{CB}_{\mathrm{L}}$ than due to the second band contributing to the transport.

Our result for $E_{\text {def }}$ is higher than the value used by Bahk et al.: $E_{\mathrm{def}, x=0.2}=8.9 \mathrm{eV}$. The difference is not due to the mobility data but rather the used elastic constant. Bakh et al. used $C_{11, x=0.2} \approx 40 \mathrm{GPa}$, i.e. a much lower value. If we use the same elastic constant we obtain $E_{\mathrm{def}, x=0.2} \approx 7.9 \mathrm{eV}$ in good agreement with their data. This disagreement can be figured out by mapping of the elastic constants with temperature and composition. Liu et al. obtained $E_{\mathrm{def}, x=0}=17 \mathrm{eV}$ and $E_{\mathrm{def}, x=1}=10 \mathrm{eV}$, which give $E_{\mathrm{def}, x=0.2}=15.4 \mathrm{eV}$ in the linear interpolation; in decent agreement with our results. ${ }^{6}$

\section{Comparison with $\mathrm{Mg}_{2} \mathrm{Si}$}

Further insight into fundamental trends can be gained by comparing the obtained material parameters of $\mathrm{Mg}_{2} \mathrm{Si}_{0.8} \mathrm{Sn}_{0.2}$ with those of the parental compound $\mathrm{Mg}_{2} \mathrm{Si}$. We have therefore plotted the relevant transport data and modeling results of a sample with composition $\mathrm{Mg}_{2} \mathrm{Si}_{0.9875} \mathrm{Sb}_{0.0125}$ in Fig. 4 and 5, labelled as " $\mathrm{Mg}_{2} \mathrm{Si}$ ". The dopant concentration and compaction parameters were optimized giving $Z T>0.7$ at $800 \mathrm{~K}$, the sample might therefore serve as a valid comparison. ${ }^{23,28,41}$ The formation of a solid solution and the observed multiphase character of the $\mathrm{Mg}_{2} \mathrm{Si}_{0.8} \mathrm{Sn}_{0.2}$ samples affect the power factor only to a small extent but reduce the lattice thermal conductivity by a factor of 2 over the whole measurement range. The general trends are not unexpected and have been observed previously. ${ }^{4,5}$ We furthermore observe a small reduction in charge carrier mobility, which is partly due to additional alloy scattering at the Sn atoms ${ }^{42}$ and partly due to the microstructure. The more fundamental question is: if and how variations in stoichiometry influence the band structure? In the model employed by Zaitsev and others, a change in the $\mathrm{Si} / \mathrm{Sn}$ ratio results in a shift of conduction band $\mathrm{CB}_{\mathrm{L}}$ and $\mathrm{CB}_{\mathrm{H}}$ with respect to each other (and the valence bands) but the curvature of the bands remains unaffected. ${ }^{4,5,20}$ Our measurement results indicate differently as we find an increase in the effective mass upon Sn substitution (see Fig. 5(b)), indicating a flattening of the $\mathrm{CB}_{\mathrm{L}}$ band. The increase in the effective mass with increase in the Sn content is in agreement with DFT results reported by Tan et $a l .{ }^{35}$ We furthermore observe a slight reduction of the deformation potential. The mean value for $\mathrm{Mg}_{2} \mathrm{Si}_{0.8} \mathrm{Sn}_{0.2}$ is $E_{\mathrm{def}, x=0.2}=13.0 \mathrm{eV}$, while we found $E_{\mathrm{def}, x=0}=15 \mathrm{eV}$ for the compound without Sn; see Fig. 5(f) and ref. 28.

Overall, the main features of the electronic transport can be well understood in the framework of a single parabolic band, although the material is not a single phase compound and has a complex microstructure. It is plausible that the matrix phase is dominant for the carrier transport as the Sn-rich phase has only a very small volume fraction $(<1 \%)$ and the $\mathrm{Mg}_{2}$ Si-like phase is significantly less doped and therefore behaves more or less like insulating particles within the matrix. The dependence of $m^{*}(T, \eta)$ indicates that the bands are not strictly parabolic. Nevertheless, the agreement between experimental data and the predictions from the simple single parabolic band model is good and the material parameters like $n_{\mathrm{opt}}, Z T_{\mathrm{max}}, m^{*}, E_{\mathrm{def}}$ can expected to be reasonably accurate. The observed deviations from the single parabolic band model point towards a nonparabolic band, rather than a contribution from the second band. This indicates a band gap between the light and the heavy conduction band of $\geq 0.2 \mathrm{eV}$, supporting an earlier work by Zaitsev et $a l .{ }^{4}$ and calculations by Liu et al. ${ }^{5}$ but in contradiction to theoretical results by Bourgeois et al. ${ }^{15}$ However, the effect of the second band cannot be totally excluded from the data. We also note that a Kane type band would lead to a lower Seebeck coefficient at high doping than a parabolic band, 
in contrast to what is found experimentally. More sophisticated modeling is therefore required for a full assessment of the band structure.

Comparing the properties of $\mathrm{Mg}_{2} \mathrm{Si}_{0.8} \mathrm{Sn}_{0.2}$ with those of $\mathrm{Mg}_{2} \mathrm{Si}$, we reveal a reduction of the phonon deformation potential and an increase in the effective mass, indicating a band flattening upon Sn substitution.

\section{Conclusions}

We have successfully synthesized $\mathrm{Mg}_{2} \mathrm{Si}_{0.8-y} \mathrm{Sn}_{0.2} \mathrm{Sb}_{y}$. The samples show a heterogeneous microstructure and a multiphase character. The main phase itself does not have one strict composition but is composed of various domains with similar compositions. We determine a maximum thermoelectric figure of merit of 0.95 at $740 \mathrm{~K}$, the best reported for this composition. The specific figure of merit is comparable to the results for $\mathrm{Mg}_{2} \mathrm{Si}_{0.4} \mathrm{Sn}_{0.6} ; \mathrm{Mg}_{2} \mathrm{Si}_{0.8} \mathrm{Sn}_{0.2}$ might thus find an application where the material weight is a crucial factor, i.e. in airborne applications. We furthermore show that the electronic transport can be modelled within a simple single parabolic model with reasonable accuracy. This allows for the extraction of fundamental material parameters like effective mass and the phonon deformation potential. Comparison with $\mathrm{Mg}_{2} \mathrm{Si}$ shows a flattening of the light conduction band, indicating that substitution of Si by Sn does not only affect the band energies but also their curvature.

\section{Acknowledgements}

The authors would like to thank W. Schönau and P. Blaschkewitz for support with the thermoelectric measurements, and A. Francke and P. Watermeyer for their help with the electron microscope. Furthermore, B. Klobes is acknowledged for useful discussion on elastic constants and K. Kelm for discussion on microstructure and phase diagrams. Special thanks to Jeff Snyder for insights into band structures and effective masses as well as to the Helmholtz Association for endorsement.

\section{References}

1 G. S. Snyder and E. S. Toberer, Nat. Mater., 2008, 7, 105-114. 2 W. Choi, S. Hong, J. T. Abrahamson, J. H. Han, C. Song, N. Nair, S. Baik and M. S. Strano, Nat. Mater., 2010, 9, 423-429.

3 S. Walia, S. Balendhran, P. Yi, D. Yao, S. Zhuiykov, M. Pannirselvam, R. Weber, M. S. Strano, M. Bhaskaran, S. Sriram and K. Kalantar-zadeh, J. Phys. Chem. C, 2013, 117, 9137-9142.

4 V. K. Zaitsev, M. I. Fedorov, E. A. Gurieva, I. S. Eremin, P. P. Konstantinov, A. Y. Samunin and M. V. Vedernikov, Phys. Rev. B: Condens. Matter Mater. Phys., 2006, 74, 045207.

5 W. Liu, X. J. Tan, K. Yin, H. J. Liu, X. F. Tang, J. Shi, Q. J. Zhang and C. Uher, Phys. Rev. Lett., 2012, 108, 166601.

6 X. H. Liu, T. J. Zhu, H. Wang, L. P. Hu, H. H. Xie, G. Y. Jiang, G. J. Snyder and X. B. Zhao, Adv. Energy Mater., 2013, 3, 1238-1244.
7 W. Liu, X. Tang and J. Sharp, J. Phys. D: Appl. Phys., 2010, 43, 085406.

8 A. U. Khan, N. V. Vlachos, E. Hatzikraniotis, G. S. Polymeris, C. B. Lioutas, E. C. Stefanaki, K. M. Paraskevopoulos, I. Giapintzakis and T. Kyratsi, Acta Mater., 2014, 77, 43-53.

9 P. Gao, I. Berkun, R. Schmidt, M. Luzenski, X. Lu, P. Bordon Sarac, E. Case and T. Hogan, J. Electron. Mater., 2013, 1-14, DOI: $10.1007 / \mathrm{s} 11664-013-2865-8$.

10 T. Dasgupta, C. Stiewe, J. de Boor and E. Müller, Phys. Status Solidi A, 2014, 1250-1254, DOI: 10.1002/pssa.201300196.

11 J.-i. Tani and H. Kido, J. Alloys Compd., 2008, 466, 335-340.

12 A. Y. Samunin, V. K. Zaitsev, P. P. Konstantinov, M. I. Fedorov, G. N. Isachenko, A. T. Burkov, S. V. Novikov and E. A. Gurieva, J. Electron. Mater., 2013, 42, 1676-1679.

13 T. Sakamoto, T. Iida, Y. Honda, M. Tada, T. Sekiguchi, K. Nishio, Y. Kogo and Y. Takanashi, J. Electron. Mater., 2012, 41, 1805-1810.

14 J. de Boor, C. Gloanec, H. Kolb, R. Sottong, P. Ziolkowski and E. Müller, J. Alloys Compd., 2015, 632, 348-353.

15 J. Bourgeois, J. Tobola, B. Wiendlocha, L. Chaput, P. Zwolenski, D. Berthebaud, Q. Recour, F. Gascoin and H. Scherrer, Funct. Mater. Lett., 2013, 06, 1340005.

16 S. F. Muntyanu, E. B. Sokolov and E. S. Makarov, Zh. Neorgan. Mater., 1966, 2, 870-875.

17 A. Kozlov, J. Grobner and R. Schmid-Fetzer, J. Alloys Compd., 2011, 509, 3326-3337.

18 L. D. Zhao, V. P. Dravid and M. G. Kanatzidis, Energy Environ. Sci., 2014, 7, 251-268.

19 W. J. Xie, J. He, S. Zhu, X. L. Su, S. Y. Wang, T. Holgate, J. W. Graff, V. Ponnambalam, S. J. Poon, X. F. Tang, Q. J. Zhang and T. M. Tritt, Acta Mater., 2010, 58, 4705-4713.

20 J. H. Bahk, Z. X. Bian and A. Shakouri, Phys. Rev. B: Condens. Matter Mater. Phys., 2014, 89, 075204.

21 W. Liu, H. Chi, H. Sun, Q. Zhang, K. Yin, X. Tang, Q. Zhang and C. Uher, Phys. Chem. Chem. Phys., 2014, 16, 6893-6897.

22 T. Dasgupta, C. Stiewe, R. Hassdorf, A. J. Zhou, L. Boettcher and E. Mueller, Phys. Rev. B: Condens. Matter Mater. Phys., 2011, 83, 235207.

23 J. de Boor, C. Compere, T. Dasgupta, C. Stiewe, H. Kolb, A. Schmitz and E. Mueller, J. Mater. Sci., 2014, 49, 3196-3204.

24 J. de Boor, C. Stiewe, P. Ziolkowski, T. Dasgupta, G. Karpinski, E. Lenz, F. Edler and E. Mueller, J. Electron. Mater., 2013, 42, 1711-1718.

25 J. de Boor and E. Muller, Rev. Sci. Instrum., 2013, 84, 065102. 26 L. J. van der Pauw, Philips Res. Rep., 1958, 13, 1-9.

27 K. A. Borup, J. de Boor, H. Wang, F. Drymiotis, F. Gascoin, X. Shi, L. Chen, M. I. Fedorov, E. Muller, B. B. Iversen and G. J. Snyder, Energy Environ. Sci., 2015, 8, 423-435.

28 J. de Boor, T. Dasgupta, H. Kolb, C. Compere, K. Kelm and E. Mueller, Acta Mater., 2014, 77, 68-75.

29 A. F. May and G. J. Snyder, in Thermoelectrics and its Energy Harvesting, ed. D. M. Rowe, CRC Press, 2012, ch. 11.

30 S. K. Bux, M. T. Yeung, E. S. Toberer, G. J. Snyder, R. B. Kaner and J. P. Fleurial, J. Mater. Chem., 2011, 21, 12259-12266. 
31 A. F. May, E. S. Toberer, A. Saramat and G. J. Snyder, Phys. Rev. B: Condens. Matter Mater. Phys., 2009, 80, 125205.

32 Y. L. Tang, Y. T. Qiu, L. L. Xi, X. Shi, W. Q. Zhang, L. D. Chen, S. M. Tseng, S. W. Chen and G. J. Snyder, Energy Environ. Sci., 2014, 7, 812-819.

33 M. I. Fedorov and V. K. Zaitsev, in CRC Handbook of thermoelectrics: Macro to Nano, ed. D. M. Rowe, CRC, Boca Raton, USA, 2006, p. 31.

34 S. Fiameni, S. Battiston, S. Boldrini, A. Famengo, F. Agresti, S. Barison and M. Fabrizio, J. Solid State Chem., 2012, 193, 142-146.

35 X. J. Tan, W. Liu, H. J. Liu, J. Shi, X. F. Tang and C. Uher, Phys. Rev. B: Condens. Matter Mater. Phys., 2012, 85, 205212.
36 D. A. Pshenai-Severin, M. I. Fedorov and A. Y. Samunin, J. Electron. Mater., 2013, 42, 1707-1710.

37 W. Zawadzki, Adv. Phys., 1974, 23, 435-522.

38 W. B. Whitten, P. L. Chung and G. C. Danielson, J. Phys. Chem. Solids, 1965, 26, 49-56.

39 L. C. Davis, W. B. Whitten and G. C. Danielson, J. Phys. Chem. Solids, 1967, 28, 439-447.

40 B. Klobes, personal communication.

41 Y. Hayatsu, T. Iida, T. Sakamoto, S. Kurosaki, K. Nishio, Y. Kogo and Y. Takanashi, J. Solid State Chem., 2012, 193, 161-165.

42 H. Wang, A. D. LaLonde, Y. Z. Pei and G. J. Snyder, Adv. Funct. Mater., 2013, 23, 1586-1596. 